\title{
Multiple Land-Use Fugacity Model to Assess the Environmental Fate of PAHs - A Mega-City Under COVID-19 Pandemic
}

\section{Qian Li}

Kyung Hee University

\section{Paulina Vilela}

Kyung Hee University

Kijeon Nam

Kyung Hee University

ChangKyoo Yoo ( $\sigma$ ckyoo@khu.ac.kr)

Kyung Hee University

\section{Research Article}

Keywords: land-use fugacity model, environmental fate, mega-city, COVID-19 pandemic, social development, soil, urban and suburban areas

Posted Date: July 26th, 2021

DOl: https://doi.org/10.21203/rs.3.rs-690113/v1

License: (9) This work is licensed under a Creative Commons Attribution 4.0 International License. Read Full License 


\section{Multiple land-use fugacity model to assess the environmental fate of PAHs}

\section{2 - A mega-city under COVID-19 pandemic}

3 Qian Li, Paulina Vilela, Kijeon Nam, Changkyoo Yoo*

4 Integrated Engineering, Dept. of Environmental Science and Engineering, College of Engineering, Kyung Hee

5 University, 1732 Deogyeong-daero, Giheung-gu, Yongin-si, Gyeonggi-do, 17104, Republic of Korea

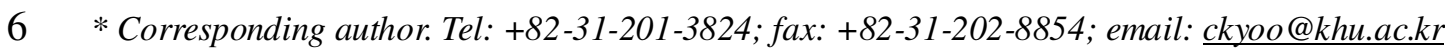

\section{ABSTRACT}

9 Along with the accelerated process of urbanization, the contradiction between social 10 development and environment become increasingly conspicuous. Herein, we access the 11 transport and fate of polycyclic aromatic hydrocarbons (PAHs) by a newly proposed multiple 12 land-use fugacity (MLUF) model to provide comprehensive understanding of PAHs 13 distribution in an urban and suburban areas. PAHs behave differently towards distinct soil 14 types and land covers within the soil phase. We suggest and implement the MLUF model to estimate the PAHs distribution in diverse land types. Moreover, we introduce a multivariate interpretation and systematic model calibration framework to validate the MLUF model

17 adaptability in different land-use area. Accordingly, the organic film on impervious surfaces holds the highest PAHs concentration than other compartments, also soil and sediment phases keep most of PAHs. The potential cancer risk of PAHs in different land-usecompartments follow the order of urban green space $>$ agricultural area $>$ forest and semi-nature area. Besides, the dynamic MLUF model is developed and implemented to the period of COVID-19 pandemic for quantitively estimating the dynamic variations of the PAHs concentration.

23 Finally, we can realize the better scientific understanding of the typical PAHs environmental pollutants transfer phenomena and distribution fates in urban and suburban areas. 


\section{Introduction}

27 The industrial revolution has brought rapid development of the economy and improved human living standards; on the other hand, social modernization has also generated severe environmental pollution issues ${ }^{1}$. Currently, many human-made pollutants have exceeded an environment's accommodating ability, and environmental contamination

31 becomes a severe concern for both humans and the overall ecosystem ${ }^{2}$. To promote the regulation of pollutants releasing and managements through the force of social laws and 33 policies, the environmental fate models that can simulate and predict pollutants 34 behaviour in the environment are getting essential for precise actions ${ }^{3}$. Significantly, chemical fate modelling is an effective way to minimize the processing time, cost, and human resources of pollutants chemicals distribution measurements ${ }^{4}$.

The fugacity-based multimedia modelling is compentent in accessing the relationship among organic pollutants physicochemical properties, environmental properties, and the chemical distribution of hazard pollution species in the environment ${ }^{5}$. Furthermore, the process complexity of the fugacity model can be adjusted regarding the various demands for providing versatile adaptability. The multimedia fugacity (MF) model is a mature technology in predicting organic chemicals' environmental behavior and fate at the global, national, and regional scales ${ }^{4,6}$. In some studies of urban systems, chemical fate models are extended beyond the typical MF model to accommodate pollutants transportation in particular environmental condition. Such as the organic films, which stand for the organic coated stationary film on a city's impervious surface area, can be considered a separate compartment ${ }^{7,8}$. Also, Song et al. ${ }^{9}$ designed a Berkeley-Trent-Urban-Rural fate model to simulate the environmental behaviour of persistent organic pollutants (POPs) by treating the air and soil compartments in urban and rural areas independently while considering the advection process in two 
51 compartments' air phase. The air and water compartments have an advective process which induces the uniform distribution of pollutants. compartments. Due to the much longer degradation time of organic pollutants in the soil phase rather than in air and water phases, pollutant concentrations can accumulate and hold significant differences among various type of soil phase ${ }^{10}$. The degradation of organic pollutants in soils are initiated by various biotic or abiotic processes, influenced by several relevant factors (like the particular environmental circumtance, unique soil properties, biota, or microbial activities) ${ }^{11}$. The time required for organic compounds degradation in the environment is enormously differ from place to place. Moreover, most researchers are roughly applying a province or city as a study area when predicting chemical distribution in a real system. It's inadequate to make any straightforward simplification of an environmental phase into a single compartment ${ }^{12}$. The different land uses and land covers are the main factors which determine the soil properties in pollutants degradation by interrupting chemicals transport processes, such as volatilization, leaching, and erosion. However, the essential transporting variations among distinct soils are not adopted in the typical MF model. To develop more efficient MF models, the versatile modeling systems construction for chemical fates assessment of pollutants is necessary.

The MF model has essential role in chemical environmental fate understanding.

71 Meanwhile, it's challenging to conduct chemical's environmental behaivors analysis 72 due to the difficulties in accurately collecting realistic model input parameters, such as 73 the chemical emission rate, compartment dimensions, and transport velocities. 74 Minimizing the difference between the calculated concentration and the actual 75 measured concentration of a pollutant is the judgment criterion for the validity of a MF 
model. A model calibration process is required to be built for estimating the performance of the multimedia fugacity model ${ }^{13}$. And the regularization or correction techniques are typically needed in the MF modelling to guarantee the solution uniqueness and stability of the models ${ }^{14}$. A statistical approach is worthy to apply in MF model for performance calibration. However, only a few studies reported the parameter calibration for an MF model before implementing models in a particular place. The chemical environmental fate modelling is particularly sensitive to environmental parameters ${ }^{15,16}$. We also develop the model parameters calibrating process to realize decent MF models which are appropriate to the specific research area. Herein, we propose a unique multiple land-use fugacity (MLUF) model which consider realistic multiple land-cover types to assess the environmental fate of organic pollutants. The MLFU model provide an efficient way to analysis the organic chemicals behaviors in the environment. In this research, a case study of polycyclic aromatic hydrocarbons (PAHs) distribution in the core districts and Tongzhou district, Beijing is investigate to verify the model performance in differentiate the diverse landcover soil phase behaviour, as the scheme illustration shown in Figure 1. Also, based on the sudden event in the world context, we conduct the dynamic MLUF model on quantitive estimate of the PAHs concentration variation under the impact of the COVID-19 pandemic. To validate and calibrate the suggested MLUF model, a multivariate statistical and system analysis framework is constructed for boosting the modelling prediction validity and efficiency. We reduce the data dimensionality through principal component analysis (PCA) and partial least squares (PLS) for visualizing and interpreting the relationships among variables. Finally, the humans and ecosystems risks posed by PAHs to are given in the study area. 


\section{Results}

102 PAH emission inventory in Beijing. The emission inventory of PAHs in Beijing was investigated and recorded for 18 years, from 2000 to 2017 , and the variation in total PAH emissions into the atmosphere is shown in Supplementary Figure S2 (a). The discharge of PAHs tended to increase during the first 18-year of 21 centuries. PAHs' emission was $427 \mathrm{t} / \mathrm{year}$ in 2000 with the domain emission source from transport

107 petroleum combustion and followed by domestic coal combustion, coke production,

108 and straw burning. The emissions in 2002 were the lowest amount recorded during the 109 period, 369 t/year. After that, PAH emissions fluctuated with a general rise trend until

110 2014. The fraction attributed to each emission source is also illustrated in

111 Supplementary Figure S2 (a). Obviously, the structure of PAH emission sources

112 changed dramatically during the study period. There are two point-in-time during the 113 investigated period that met the changes in emission structure. The first one was in 114 2011, coke production was prohibited in Beijing, and the second time point was in 115 2015, the straw burning was restricted in the whole Beijing city. Transportation 116 releasing was the dominant source of PAHs in Beijing during the study period, a 117 common phenomenon in megacities. Supplementary Figure S2 (b) shows the 118 contribution of PAHs with different ring numbers to the total PAH emissions in 2010.

119 It is noticeable that PAHs with a low number of rings (2-ring and 3-ring) compose a

120 large fraction of the total PAH emissions, $40 \%$ and $35 \%$ for PAHs with 2 rings and 3 121 rings, respectively. PAHs with high molecular weights (4, 5, and 6 rings) make up 25\% 122 of all PAH emissions.

123 In this study, the gross domestic product (GDP) ratio obtained from Beijing 124 Statistic Yearbook between the study area (RMB 92.32 billion) and Beijing city 125 (RMB 141.14 billion) in 2010 was used to estimate the emission inventory of PAHs 
126 due to the potential proportional relationship among GDP and PAHs release ${ }^{17}$.

127 Therefore, the total PAH emission rate in the study area was $33.73 \mathrm{~kg} / \mathrm{h}$ in 2010 and

128 this emission rate is used to establish the MLUF model and the calibration of this

129 model.

130

131 Principle component analysis. Eighty-five parameters of the MLUF model with three

132 different soil compartments were selected and analyzed and these parameters are

133 summarized in Supplementary Table S4. The multivariable results for the output

134 model concentration and the input model parameters implemented by PCA were used

135 to interpret the relationships between those two groups of variables. Characteristics

136 grouped all the MLUF model parameters to identify interactions with the

137 concentration variable, which included chemical properties, environmental properties,

138 compartment size, and mass transfer coefficients (the detail of each parameter is

139 shown in Supplementary Table S3). A visible representation of the first three principal

140 components is shown in Supplementary Figure S3. And Supplementary Figure S3 (a)

141 shows the relation between chemical properties and concentrations. The PAH

142 concentrations in air and vegetation are close to each other and give highly positive

143 contributions to the first principle component (PC1). On the negative side of PC1,

$144 \operatorname{logKoa}$ and the concentration in the organic film have considerably high values,

145 which indicates that $\operatorname{logKoa}$ (as a thermodynamic descriptor between the air and

146 organic phases) positively affects $C_{F}$ and negatively influences $C_{A}$. Also, $C_{V}$ shows

147 the same tendency with $\log$ Koa as $\mathrm{C}_{\mathrm{A}}$ because the respiration of plants makes a large

148 connection between $\mathrm{C}_{\mathrm{V}}$ and $\mathrm{C}_{\mathrm{A}}$. The concentrations in soil, sediment, and water are the

149 main factors contributing to the second principal component (PC2) and can be treated

150 as a solubility factor. Meanwhile, $\operatorname{logKoc}$ and $\operatorname{logKow}$ make larger contributions to the 
151 soil and sediment factors than the other model parameters. Additionally, the half-life

152 degradation time has a high correlation with a concentration in the corresponding

153 phases. Supplementary Figure S3 (c) shows the relationship between compartment

154 sizes and concentrations. It clearly shows the negative association between

155 concentration and the corresponding compartment sizes. The points of concentration

156 in air and organic film are in the opposite direction with the leaf area index because

157 the absorption effect of plants causes $\mathrm{BaP}$ to attach to leaves, which reduces the 158 concentration of PAHs in the air and organic film.

160 Key parameters selection via PLS-VIP. The environmental characteristics of the

161 various parts of the study area work in synergy with the inherent physical and 162 chemical properties of the PAHs to influence their distribution and ultimate 163 environmental transport and fate. Supplementary Figure S4 illustrates the V-plot 164 obtained by implementing the MLUF model on BaP. The V-plot presents the 165 PLS-derived VIP scores versus the correlation coefficient value for all model 166 parameters. It should be pointed out that correlation coefficients have an obvious 167 disadvantage: the degree to which they approach 1 is related to the number of data 168 sets (n). When $\mathrm{n}$ is small, the correlation coefficient fluctuates greatly, and the 169 absolute value of the correlation coefficient for some samples easily approaches to 1 ; 170 when $n$ is large, the absolute value of the correlation coefficient tends to be small.

171 Therefore, it is inappropriate to judge that variables $\mathrm{x}$ and $\mathrm{y}$ have a close linear 172 relationship based only on a large correlation coefficient between them. The VIP 173 values show the influence of each model parameter on the model output, and any 174 parameter with a score greater than 1 is generally considered to be important. In the 175 present analysis, the correlation coefficient quantifies the degree of linear correlation 
between the model parameters and the model output (concentrations). The emission rate $\left(E_{a}\right)$ has a larger positive correlation with the model output than with the other parameters. The half-life degradation in the air $\left(\mathrm{hf}_{\mathrm{a}}\right)$ keeps the second-largest effect on the model concentrations. It is evident that the $\mathrm{hf}_{\mathrm{a}}$ reflects the decomposition rate of $\mathrm{BaP}$ in air, and the air is the only phase with $\mathrm{BaP}$ emissions; therefore, to a certain extent, the $\mathrm{hf}_{\mathrm{a}}$ determines the quantity of $\mathrm{BaP}$ that remains in the air for potential transport to other phases. As shown in Supplementary Figure S4, besides Ea and $\mathrm{hf}_{\mathrm{a}}$, parameters related to the partition coefficient (logKoa, $\log$ Kow, and $\log K o c$ ) and the half-life degradation times in soil and sediment also relative highly influenced the model output on BaP.

The VIP scores of each PAH are summarized in Figure 4. The model parameters with VIP scores lower than 1 are shown in dark blue indicated that these parameters have a minor influence on the simulated concentrations. $E_{a}$ (parameter 16) and Qain (or Qaout, parameter 82, 83) had the largest VIP value among the model parameters for all 16 PAHs. As illustrated above, the 85 model parameters were defined in four groups: chemical parameters (parameter 1 to 15), environmental parameters (parameter 16 to 35), compartment sizes (parameter 36 to 48), and mass transfer coefficients (parameter 49 to 85 ). The chemical parameters show larger VIP values than the other parameters. The partition coefficients (logKow, logKoa, logKoc, corresponding to parameter 3, 4, and 5) parameters are influential for the model output of all PAHs. Those parameters are related to the partitioning behavirors of the pollutants, a relative indicator of the tendency of an organic compound in one phase to adsorb to another. $\mathrm{E}_{\mathrm{a}}$ and $\mathrm{hf}_{\mathrm{a}}$ show a high VIP score of all pollutants which is due to the emission sources of PAHs is mostly released into air phase. And the hf $\mathrm{f}_{\mathrm{a}}$ governs the amount of pollutants transport to the other phases to a large extent. Due to the 
201 sluggishness property of soil and sediment, these compartments are like the universal 202 sink of pollutants and the pollutants can easily accumulate. The degradation is an 203 important process to remove pollutants in soil and sediments, therefore, the $\mathrm{hf}_{\mathrm{s}}$ and $204 \mathrm{hf}_{\text {se }}$ have relatively high VIP scores in the MLUF model. To sum up, the model 205 parameters of $\operatorname{logKow}, \operatorname{logKoa}, \operatorname{logKoc}, \mathrm{hf}_{\mathrm{a}}, \mathrm{hf}_{\mathrm{s}}$ of three land-use soil, and $\mathrm{hf}_{\mathrm{se}}, \mathrm{h}_{\mathrm{a}}$, 206 were selected as the input variables for the GA to calibrate the model concentrations 207 to the study area.

209 Estimated environmental fate of PAHs via MLUF model. The calibrated 210 concentrations were compared with observed PAH concentrations to verify the model 211 performance. The total PAH concentration and PAH equivalent concentrations are 212 illustrated in Supplementary Figure S5 (a) and (b), respectively. The PAH equivalent 213 concentration was derived by considering the toxicity of each chemical and 214 multiplying the concentration of each PAH by a toxicity equivalence factor (TEF). 215 The simulated (bar plot) and observed concentrations (the maximum, mean, and 216 minimum values are shown as red points) were compared in each environmental 217 compartment, air $\left(\mathrm{ng} / \mathrm{m}^{3}\right)$, water (ng/L), soil (ng/g), sediment (ng/g), vegetation (ng/g), 218 and organic film $\left(\mathrm{ng} / \mathrm{m}^{2}\right)$. The model-estimated total PAH concentrations and the 219 equivalent PAHs concentration are consistent with the measurements (within the 220 lower and upper boundaries of the reference concentrations). Therefore, the proposed 221 MLUF model can be conducted to predict the environmental fate of PAHs. The 222 calibrated parameters were also implemented in a typical MF model (single soil 223 compartment) to compare the results with those from multiple soil compartments. The 224 calculated PAH concentrations (in 2010, same year base with MLUF) of MF model 225 which consider soil as single compartment at the study areais shown in 
Supplementary Figure S6, and the results show that the calibrated parameters are

227 suitable for estimating the PAH distribution in the study area. The estimated total

228 PAH concentrations in the MF model and the proposed MLUF model are compared in

229 Supplementary Table S11. Because the environmental compartments in these two

230 models varied, the estimated concentrations vary slightly in each compartment. The

231 PAH concentrations calculated by the MLUF model in the soil compartments and 232 vegetation under different land uses shown the highest value in city gardens and the 233 lowest value in the FSNA. The single-compartment (soil or vegetation) PAH 234 concentrations are in the same range as the multiple-compartment PAH 235 concentrations. Among the evaluated PAH concentrations, the PAH component 236 patterns differed among each compartment (Supplementary Figure S7 (a)). The low 237 molecular weight (LMW, less than four benzene rings) PAHs appear abundantly in 238 the air and water phases because of their relatively high vapor pressure and water 239 solubility. In other compartments (soils, sediment, vegetation, and organic film), high 240 molecular weight (HMW, more than three benzene rings) PAHs are predominant 241 because they partition more readily into organic matter. PAHs with a large TEF value 242 contribute more than other PAHs to the $\mathrm{PAH}_{\text {seq }}$ concentration (Supplementary Figure 243 S7 (b)).

244 In the study area, 295.45 tons of PAHs were released into the air compartment in 2452010 and distributed to other compartments through portioning and transport 246 processes. An equilibrium state can realize according to the theories in the 247 steady-state fugacity model. The mass balance diagram of the $\sum_{16} \mathrm{PAHs}$ fate and 248 transport processes is shown in Figure 5, with a total $\sum_{16} \mathrm{PAH}$ emission rate in the 249 atmosphere of $33.73 \mathrm{~kg} / \mathrm{h}$. Once PAHs have been released into the ambient atmosphere, 250 most of them are transport through advection $(121.39 \mathrm{~kg} / \mathrm{h})$. Due to the short half-life 
251 degradation time in air, $31.8 \mathrm{~kg} / \mathrm{h}$ PAHs were removed from air compartment through

252 degradation. The $5.72 \%$ portion PAHs in air phase transports to other compartments.

253 Among those remaining PAHs, HMW PAHs tend to behave like solid particles in the 254 atmosphere. Due to the adsorption of particulate matter by plants, most of the air phase 255 remaining PAHs were deposited into vegetation (with $4.36,8.38$, and $1.56 \mathrm{~kg} / \mathrm{h}$ for 256 agriculture, FSNA, and urban green space, respectively). Only $0.21 \%$ of PAHs dissolve 257 in the water compartment. Meanwhile, $82.28 \%$ of PAHs were removed by the reactions, 258 and 5.94\% were transferred to other cities through the air. At equilibrium, most released 259 PAH pollutants in air are removed by responses in the other four compartments, 260 consisting of $93.77 \%$ of all the discharged PAHs in the study area. The remaining $7.23 \%$ 261 of PAH pollutants were found enriched in the soil phase. In other words, the soil phase 262 holds $95.69 \%$ of all remaining PAHs in environments. When considering the volume of 263 the phases, the PAHs concentration in the organic film $\left(6.19 \times 10^{3} \mathrm{mg} / \mathrm{m}^{3}\right)$ is highest, and 264 the second-highest case is the city green space soil-type phase $\left(3.90 \times 10^{3} \mathrm{mg} / \mathrm{m}^{3}\right)$.

The effect of land-use changes on the environmental fate of PAHs. The calibrated MLUF model was implemented to predict the PAH distributions in 2015 and 2020 by projecting the various emission rates and land-use areas within the overall study area. In 269 this study, the PAH emissions in 2020 regards the same as 2015 by considering the city 270 development has slowed down and the PAHs emission source structure tending towards 271 the stable. The land-use areas between 2015 and 2020 have changed according to 272 municipal planning with keeping the area of urban construction land and improving the 273 ecological land use. The land-use (agriculture, city green area, water area) of the study 274 area in 2020 is estimated via the linear regression model based on the general plan for 275 land use and land-use changes in Beijing from 2012 to 2017, in which the land-use data 
from the Beijing statistical yearbooks (as shown in Supplementary Figure S8). The

277 modelled multicompartment $\Sigma_{16}$ PAHs concentrations show higher value in 2010 278 compared with 2015 and 2020 (expect water and sediment compartments) (Table S12).

279 Compared to the land-use change between 2015 and 2020, the areas of farmland, urban 280 green space, and water are reduced by $4.69 \%, 8.22 \%$, and $5.04 \%$, respectively. The 281 FSNA area is increased by $8.54 \%$ and the urban construction area is increased by $0.62 \%$. 282 The $\Sigma_{16}$ PAHs concentration in water and sediment compartments shown a rising trend. 283 There are two possible reasons to cause this phenomenon, one is due to the water area is 284 descending and the second is the increase of urban construction area induced more 285 PAHs transfer from organic film to water compartment. In the air, vegetation, and 286 organic film compartments, the $\Sigma_{16} \mathrm{PAHs}$ concentration had slightly increased. The 287 increase of FSNA caused the $\Sigma_{16} \mathrm{PAHs}$ concentration have a $0.02 \%$ decrease in FSNA 288 soil. The mass of PAHs in the various environmental compartments depends on the 289 concentrations and compartment sizes. The changes in the land-use area also directly 290 influence the PAHs mass content in each compartment. The PAHs in water, sediment 291 compartments are decreased, on the contrary show increasing in air and organic film compartments. The land-use changes in soil compartments lead to similar changes 293 happened in the soil and vegetation compartments. The soil compartments area 294 changed by $-4.69 \%, 8.53 \%$, and $-8.15 \%$, corresponding to agriculture soil, FSNA soil, 295 and city green area. Through this MLUF mode, the pollutants dynamic distribution 296 responses along with the city developments could be performed for subsequently 297 providing scientific guidance and adequate actions to environmental regulation.

299 Risk assessments. Risk assessments about the environmental PAHs were carried out 300 for both human health and ecological systems under the 2010 case, and the results are 
301 shown in Figure 6, where the red points stand for the total risk posed by the 16 PAHs, 302 and the stacked bar chart shows the contribution of each PAH to the total risk. Figure 3036 (a) expresses the human ILCR values retained by PAHs in different areas through 304 various exposure routes. In this study, inhalation was the dominant route of human 305 exposure to PAHs, followed by dermal contact and ingestion. The ILCR values for 306 different land cover regions with different land cover followed the urban green space > 307 agricultural > FSNA, indicating that the urban green space soil is more hazardous than 308 agriculture soil and FSNA soil. The ILCR exposure by 5 rings PAHs occupy more than $30985 \%$ of the total ILCR through all exposures routes due to the higher health risk 310 pertained by the 5 rings PAHs than the other PAH species. Therefore, the emission rates 311 of the PAHs are not the principal element that influences health risk. Even though the 312 LMW PAHs have the highest emission rates, but their risk just occupies less than $2 \%$ of 313 the total ILCR. As a consequence, more attention should be assigned to the fates and 314 distribution of the high ILCR PAHs species in soil from society.

315 The risk assessment for the ecological systems of water, soil, and sediment estimated 316 hazard values are shown in Figure 6 (b). In the study area, the sediment and water 317 phases have higher ecological risk, with a value of 1.74 and 1.50 , respectively, 318 indicating that the PAHs released in the study area pose a hazard to aquatic organisms. 319 The HMW PAHs show more substantial contributions to the RI value in the water phase. 320 The RI in the soil compartments was lower than the limit values, indicating that those 321 PAH concentrations are within the acceptable range in the soil compartment of three 322 land-use. Compared with the RI in the water phase, LMW PAHs accounted for a larger 323 proportion of the risk by PAHs in sediment and soil ecosystems. The RI value for 324 different land covers followed the order of urban green space > agricultural area > 325 FSNA, indicating that the soil in urban green space is more hazardous to the ecological 
system than agricultural soil and FSNA soil. The contribution of each PAH to the RI

327 also differs by land cover. In agricultural soil, the HMW PAHs (5 and 6 rings) account

328 for more than $77 \%$ of the total PAHs ecological risk, show $66 \%$ and $61 \%$ in FSNA and

329 urban green space, respectively. The results of this study have significant implications

330 for urban planning and decision making when working to protect and remediate water

331 and habitat quality in Beijing, also the assessment techniques which described here

332 are applicable to other study areas.

333 The spatial distribution of PAHs caused ILCR is shown in Figure 7. The land use 334 information of the study area is extracted based on the Beijing Land Use Planning 335 (2006 - 2020) and Wanghe et al. ${ }^{18}$. Continue PAHs posed healthy risk were predicted 336 by interpolation with the ILCR estimated by MLUF model and health risk model 337 through multiple exposure routes in different land-use using the inverse distance weight 338 method in ArcGIS 10.5. Any substance with an ILCR value higher than $10^{-6}$ and lower 339 than $10^{-4}$ is considered to be potentially carcinogenic ${ }^{19}$. The study area has been 340 exposed to excessive PAHs and could therefore face high potential health risks. In the 341 study area, people PAHs exposure health risk is higher in the central city area of core 342 districts in Beijing and Tongzhou district. As a new urban development area in Beijing, 343 Tongzhou district is dominated in an agricultural area with $37.6 \%$ rather than the $5.2 \%$ 344 in core districts. Attributed to the high agricultural area percentage of Tongzhou district 345 than Beijing core districts, the ILCR caused by PAHs in Tongzhou district is lower than 346 the core districts. With the land-use information, the MLUF model integrates with risk 347 model can effectively distinguish the pollutants concentration distribution in soil 348 phases. 
351 the study area during the COVID-19 pandemic. The dynamic MLUF model is 352 implemented for the first four months in 2020, which covered the epidemic outbreak 353 and got under control in Beijing. The emission of PAHs from transport is estimated by 354 the travel intensity index in Beijing, as shown in Supplementary Figure S10 ${ }^{20}$. 355 According to the travel intensity index, a trafficking factor is calculated to reflect the 356 traffic volume reduction due to the restriction and control measures. Four stages are 357 considered according to the dataset. On the first day of 2020, WHO had set up the IMST 358 (Incident Management Support Team) to dealing with the COVID-19 outbreak. 359 Therefore, the early stage is defined as regular travel, which is the period before the 360 Chinese lunar New Year and the time that the majority of people knew nothing about 361 this new coronavirus. As the Lunar New Year approaching, some migrant workers were 362 started to return to their hometown after January $18^{\text {th }}$, and the inner-city travel intensity 363 index had been steadily reducing, as shown in Supplementary Figure S10, which 364 continued for around one week as considering the second stage. At the end of the 365 second stage, the news of human-to-human transmission of novel coronavirus has been 366 reported and known by the most public. The widespread news of the novel coronavirus 367 coming out leads to a lower inner-city travel intensity index than in the same period of 368 2019, Supplementary Figure S9 in Supplementary Information (SI). The third stage is 369 home quarantine, which from January 26th to February 9th. In this period, the traffic in 370 Beijing city remains at a low level compared with other years. The fourth stage is a step 371 by step return to work since February 10th. The linear piecewise functions are 372 developed to describe the traffic factor during this period, as shown in Eq. (9).

$373 \quad f_{\text {traffic }}=\left\{\begin{array}{lc}1, & t \leq 18 \\ -0.11 t+1, & t \leq 25 \\ 0.23, & t \leq 40 \\ 0.0056 t, & 40<t \leq 121\end{array}\right.$ 
374 where $t$ stands for the day counting start from January $1^{\text {st }}$ to April $30^{\text {th }}$, and $f_{\text {traffic }}$ is the 375 traffic factor. The PAHs released from transportation are calculated by multiply the 376 traffic factor with the estimated transport PAHs emission in 2020. As shown in 377 Supplementary Figure S10, the traffic factor calculated by the simple linear regression 378 can predict the traffic volume trend during the epidemic period.

379 The dynamic MLUF model estimates the concentration of $\Sigma_{16} \mathrm{PAHs}$ in the first four 380 months of 2020, and the concentration in each compartment are shown in Figure 8. Due 381 to the reduction of emission, the total PAHs concentration is shown a decline during this 382 period. The overall decreasing rate of total PAHs concentration is calculated with the 383 initial level and the concentration at the end of the analysis period summarized in Table 384 S13. PAHs concentration in water phases presents the most significant changes. 385 However, in soil and sediment phases, the $\Sigma_{16} \mathrm{PAHs}$ concentration shows fewer changes, 386 indicating that the PAHs are more stable in these phases than air, water, vegetation, and 387 organic film due to the longer degradation time. During this period, the PAHs emissions 388 go through dramatic decreases to a relatively lower level and rise gradually. However, 389 the $\Sigma_{16}$ PAHs concentration in soil and sediment phases has a minor reflection of the 390 decline in emission, as shown in Figure 8 (c-f). The predicted concentration is 391 monotonically decreasing in this period. The emission changes show the most 392 considerable influence on the concentration in the air phase. The $\Sigma_{16} \mathrm{PAHs}$ 393 concentration in the air decreased by $51.59 \%$ compared with the concentration before 394 the COVID-19 epidemic due to the home quarantine in stages 2 and 3. It gradually 395 increased by $63.61 \%$ compared to the lowest concentration along with the step-by-step 396 restoration of the economic activity. Due to the lower degradation time and advection 397 rate compared with the air phase, the $\Sigma_{16} \mathrm{PAHs}$ concentration in water shows slightly 398 smaller change, reducing $48.35 \%$ in stages 2 and 3 and increasing $54.75 \%$ in stage 4 . 
399 For the vegetation and organic film compartments, the $\Sigma_{16} \mathrm{PAHs}$ concentration is 400 sensitive to the variation of emission due to the close relations with the air phase.

401 Considering the land-use in the study area, the vegetation compartment in the city green 402 space is more sensitive to the PAHs emission changes than the other lands. The PAHs 403 concentration in the city soil compartment is more stable than the different land-use 404 with a reduced rate of $2.64 \%$. The concentration reduction rate differs in various 405 land-use indicated that the PAHs pollutants in city soil are hard to remove by natural 406 degradation processes.

\section{Discussion}

409 A steady-state MLUF model was proposed and successfully implemented to 410 transferring dynamics and chemical fate estimation of PAHs pollutants in Beijing, 411 China by considering the soil phase variances to several detailed realistic 412 compartments. The MLUF modelling process was calibrated by multivariate 413 interpretation and systematic model, and reached comprehensive validation. And we 414 selected the essential model parameters of MLUF through the unique approach of 415 PLS-derived VIP scores versus the correlation coefficient values. We acquired the 416 spatial distributions of the 16 kinds of PAHs species in soil phases of the study area 417 from the MLUF model. The PAHs movement and transformation properties inside the 418 compartments were determined for representing the estimated mass transport rates. 419 After performing the risk assessment, we first time obtained the ILCR health risk 420 values in the different land covers that follow the urban green space > agricultural area > 421 FSNA. In terms of ecological risk, the RQ value for different land covers followed the 422 same sequence with ILCR case, indicated the more hazardous risk possessed in urban 423 green space soil than agricultural and FSNA soil. Interestingly, we observed the 
obvious concentrations changes quantitively during the COVID-19 pandemic in the

425 study area through implementing the proposed dynamic MLUF model. The significant 426 influence of the COVID-19 outbreak on PAHs concentration was discovered in the air, 427 water, vegetation, and organic film compartments. This study provides a throughly 428 scientific understanding of environmental pollutant transport in urban areas with the 429 most recent environmental and social information and can be applied to facilitate 430 various land conservation and sustainable development efforts.

\section{Methods}

Model description. The MLUF model is established based on an MF Level III model to evaluate the pollutant fates in six main compartments, i.e., air, water, soil, sediment, vegetation, and impervious surfaces (organic film), shown in Supplementary Figure S1. The air compartment consist of gas and particulate matter. The water compartment consists of water and particulate matter. The sediment compartment is regarded as a layer of deposited particles at the bottom of the water body. In the urban environment, impervious surfaces, such as buildings and roadways, make up a large 440 proportion of the downtown area, impeding the mass transfer between air and soil 441 compartment. Especially, an organic film coated on the impervious surfaces is considered as a single compartment in the strategy of MLUF model. The absorbed organic pollutants was considered mainly migrates from impervious surfaces to water compartment rather than soil compartment through rain wash-off into urban drainage system. As one of the unique aspect in this report, the soil compartment in MLUF 446 model is splited into several independent sub-compartments to be more realistic and 447 each soil sub-compartment is considered to carry independent transport behaviors of 448 pollutants compounds with other compartments. The separation of soil compartment 
449 is based on the land-cover types. According to different land cover, the vegetation 450 compartment is seperate to different sub-compartments. The mass-balance equation in 451 each compartment of the MLUF model assumes that the total input of chemicals is 452 equal to the sum of the total output and the accumulation, as following:

$E_{i}+Q_{i n} \cdot C_{i}+\sum\left(D_{j i} f_{j}\right)-f_{i}\left(\sum D_{i j}+D_{A d i}+D_{R i}\right)=\alpha$

454 where $E(\mathrm{~mol} / \mathrm{h})$ is the emission rate, $D(\mathrm{~mol} / \mathrm{Pa} \cdot \mathrm{h})$ is the transport parameter, $f(\mathrm{~Pa})$ is 455 the fugacity of chemicals, $C\left(\mathrm{~mol} / \mathrm{m}^{3}\right)$ is the concentration of the pollutant, and $Q_{\text {in }}$ $456\left(\mathrm{~m}^{3} / \mathrm{h}\right)$ is the advection flow rate of air and water. The subscript $A d$ represents the 457 adventive process, $R$ is the reaction, and $i$ and $j$ represent environmental compartment 458 numbers. On the right side of the equation, $\alpha$ interpreted as 0 in the steady-state model 459 and $V_{i} Z_{i} \cdot d f / d t$ in a dynamic model, respectively. The steady-state mass balance 460 equations of chemicals in each compartment are summarized in Table 1. Subscripts A, $461 \mathrm{~W}, \mathrm{~S}, \mathrm{Se}, \mathrm{V}$, and F are defined as air, water, soil, sediment, vegetation, and organic film, 462 respectively. For different soil and vegetation compartments, $\mathrm{n}$ is used to distinguish 463 different sub-compartments. The chemicals concentration of each compartment is 464 computed by multiple fugacity capacity with the fugacity. The details of computing 465 fugacity capacities (Z) and transport processes (D) are shown in Tables S1 and S2.

Systematic model calibration framework for MLUF model. Figure 2 illustrates the framework for the systematic model calibration of the MLUF model using a 469 multivariate statistical analysis method. In this study, PCA and PLS were 470 implemented to find the relationships among parameters and select the critical 471 parameters that would allow the proposed model to precisely predict the environmental behavior of organic pollutants. Firstly, the process of predicting the 
model. At second, the Monte-Carlo simulation method was applied to the fugacity model with a random combination of model parameters to obtain an experimental data set. Thirdly, auto-scaling was used in data preprocessing to normalize the data. Then, using PCA and PLS, the parameters were grouped, and sensitive variables were investigated. A system analysis of the MLUF model was conducted for the affected variables to identify how those parameters influence the model output. Such a model calibration is required before an MF model can be applied to predict chemical behavior. The PAH concentrations measured in each compartment were gathered from the literature and compared with the concentrations calculated in the model calibration process. In the end, a genetic algorithm (GA) approachwas used to optimize the model parameters by minimizing the deviation between the modelled and observed PAH concentrations. The description of systematic model calibration methods are shown in SI, and the MLUF model parameters are listed in Supplementary Table S3 and S4.

\section{Case study}

PAHs. As a class of persistent contaminants, PAHs have a relatively long degradation time in the environment, are easily diffused and able to travel a long way in the environment. Especially, PAHs are an increasing concern because they are highly toxic to humans and ecological systems. In this study, 16 priority ordinary PAHs were selected basing on the potential human and ecological health effects according to the claim of U.S. Environmental Protection Agency (USEPA): naphthalene (NAP), acenaphthylene (ACY), acenaphthene (ACE), fluorene (FLU), phenanthrene (PHE), anthracene (ANT), fluoranthene (FLA), pyrene (PYR), benzo[a]anthracene (BaA), chrysene (CHR), benzo[b]fluoranthene

$(\mathrm{BbF})$, benzo[k]fluoranthene $(\mathrm{BkF})$, 
benzo[a]pyrene (BaP), indeno[1,2,3-cd]pyrene (IcdP), dibenzo[a,h]anthracene (DBA), and benzo[ghi]perylene (BghiP). Their physicochemical properties are summarized in

501 Supplementary Table S5 ${ }^{21}$.

502 PAHs are released into the ambient environment by several natural and

503 anthropogenic processes ${ }^{22}$. Since the second industrial revolution, PAHs'

504 human-caused emissions have became substantial because of the rapid increase in 505 burned fuel for industrial manufacturing, transportation, and heating applications ${ }^{23}$. An 506 elaborate emission inventory needs to be estimated to establish an accurate model for 507 predicting PAHs' environmental behavior. In Beijing, the following PAHs emission 508 sources were identified: (1) industrial coal combustion, including thermoelectric power 509 stations and heating plants; (2) domestic coal combustion, including the residential and 510 commercial sectors; (3) coke production; (4) transport petroleum combustion; (5) other 511 petroleum combustion; (6) straw burning; and (7) cooking. The total emission of PAHs 512 is the accumulation of the emission inventory from each source. The emission 513 inventory $(Q)$ of each chemical from each release source is calculated according to the 514 emission factor $(E F)$ and emission activity $(E A)$, as shown in Eq. (8).

$515 Q=E A \times E F$

516 The emission factors differ among PAH species and emission sources, and they are 517 listed in Supplementary Table S6. According to these reported data in Supplementary 518 Table S6, it can be observed that the industrial coal combustion process produced less 519 PAH than domestic discharge (domestic coal combustion, straw burning, and cooking) 520 which may attribute to the more adequate combustion process. Straw burning has the 521 most considerable unit-emission rate. Due to China's particular cooking style, the PAHs 522 released during cooking are also considered in this study (Eq. S7). The detailed 523 calculation of PAHs emission inventory from each emission source is shown in SI. 
525 Study area. Beijing is the capital city of China and plays an important role as the 526 country's political, economic, and cultural center. And, Beijing is located in the 527 Northern part of China $\left(116^{\circ} 20^{\prime} \mathrm{E}, 39^{\circ} 56^{\prime} \mathrm{N}\right)$ (Figure 3), and it has been experiencing 528 a relative air pollution concern continually. Besides, Beijing typically has a warm, 529 temperate, sub-humid continental monsoon climate, and it is surrounded by the Yan 530 Mountains and Loess Plateau on three sides. Pollution easily becomes trapped and 531 accumulates in this area due to weak atmospheric ventilation ${ }^{24}$. The geographical 532 impact, industrialization, and urbanization are the important causes of the pollution in 533 Beijing and its adjacent areas. As the city's environmental problems become a more 534 widespread concern, environmental protection measures have been put forward, and 535 more stricter environmental rules have been enforced during the past decades.

536 The study area consists of six core districts and Tongzhou district of Beijing, which 537 is a typical urban and suburban area. According to the Beijing Municipal Bureau 538 statistics in $2012,58.7 \%$ of the study areas are urban and townland, which is assumed 539 as artificial impervious surfaces in the MLUF model of this study. The remaining areas 540 are agricultural land (18.4\%), forest and semi-nature area (FSNA) (13\%), and urban 541 green space (3.6\%). All those land types comprise the multiple land-use parts of this 542 study. Supplementary Table S8 shows the compartment dimensions of the study area.

544 Risk assessment. As a group of hazardous POPs, PAHs have potential or identified 545 carcinogenicity and genotoxicity ${ }^{25}$. Therefore, it is cardinal significant to implement a 546 thorough toxicity analysis of PAHs. In this study, the incremental lifetime cancer risk 547 (ILCR) is applied to estimate the potential human health risk caused by the 16 PAHs. 548 People exposed to PAHs from the ambient air, water, and soil is mainly through three 
exposure routes, including dermal contact, inhalation, and ingestion ${ }^{21}$. Moreover, the related ecological risk of the PAHs in the study area are also evaluated to present a

551 comprehensive understanding of the pollutants risks. Ecotoxicity endpoint tests, such 552 as $\mathrm{LC}_{50}$ (the concentration of a chemical at which $50 \%$ of the test organisms die), $553 \mathrm{LD}_{50}$ (median lethal dose), and NOEC (no observed effect concentration), are 554 conducted to determine the level of a substance's hazardous effects to the 555 environmental compartments. To assess the ecotoxicity of a chemical, a risk quotient 556 (RQ) is suggested by the USEPA, which is calculated based on the dose of exposure 557 and ecological effects data ${ }^{26}$. In this study, the chronic risk is used to estimate the 558 ecological risk posed by PAHs. The estimated concentration of each PAH in the 559 ecosystem (soil, water, and sediment) was divided by its effect toxicity value to derive 560 an RQ, and the risk index (RI) in each compartment for all PAHs is calculated by the 561 sum of the RQ of each PAH. The details of the risk assessment equations are shown in 562 SI. The parameters of the human health risk model and ecological risk model are 563 shown in Supplementary Table S9 and S10.

565 Acknowledgments

566 This work was supported by the National Research Foundation of Korea (NRF) grant 567 funded by the Korean government (MSIT) (No. 2021R1A2C2007838) and the 568 Korea Ministry of Environment (MOE) as a Graduate School specialized in Climate 569 Change.

570 Authors' contributions

571 Q. L.: Conceptualization, Methodology, Validation, Writing original draft. P. V.: Formal 572 analysis. K. N.: Formal analysis. C. Y.: Conceptualization, Supervision, Review \& 573 Editing, Funding acquisition. 


\section{Competing interests}

575 The author(s) declare no competing interests.

\section{Ethics declarations}

577 This article does not contain any studies involving human, animal, or plants participants 578 performed by any of the authors.

\section{References}

581 1. Misra, V. \& Pandey, S. D. Hazardous waste, impact on health and environment for development of better waste management strategies in future in India. Environ. Int. 31, 417-431 (2005).

2. Morrissey, A. . \& Browne, J. Waste management models and their application to sustainable waste management. Waste Manag. 24, 297-308 (2004).

3. MacLeod, M., Scheringer, M., McKone, T. E. \& Hungerbuhler, K. The state of multimedia mass-balance modeling in environmental science and decision-making. Environ. Sci. Technol. 44, 8360-8364 (2010).

4. Su, C., Zhang, H., Cridge, C. \& Liang, R. A review of multimedia transport and fate models for chemicals: Principles, features and applicability. Sci. Total Environ. 668, 881-892 (2019).

5. Cao, X. et al. Source emissions and climate change impacts on the multimedia transport and fate of persistent organic pollutants, Chaohu watershed, eastern

595 6. MacLeod, M. et al. BETR global - A geographically-explicit global-scale multimedia contaminant fate model. Environ. Pollut. 159, 1442-1445 (2011). of chemical dynamics in an urban area. Chemosphere 44, 1655-1667 (2001). 
599 8. Domínguez-Morueco, N. et al. Application of the Multimedia Urban Model to 600 estimate the emissions and environmental fate of PAHs in Tarragona County, 601 Catalonia, Spain. Sci. Total Environ. 573, 1622-1629 (2016).

602 9. Song, S. et al. Urban and rural transport of semivolatile organic compounds at 603 regional scale: A multimedia model approach. 39, 228-241 (2016).

604 10. Liu, Y. et al. Air-soil diffusive exchange of PAHs in an urban park of Shanghai 605 606 based on polyethylene passive sampling: Vertical distribution, vegetation influence and diffusive flux. Sci. Total Environ. 689, 734-742 (2019).

11. LIAO, M. \& XIE, X. Effects of combination of plant and microorganism on 608 degradation of simazine in soil. J. Environ. Sci. 20, 195-198 (2008).

609 12. Song, S. et al. Urban and rural transport of semivolatile organic compounds at 610 regional scale: A multimedia model approach. J. Environ. Sci. (China) 39, 228-

612 13. Kim, Y. S., Kim, M. H. \& Yoo, C. K. A new statistical framework for parameter 613 subset selection and optimal parameter estimation in the activated sludge model.

615 14. Donoho, D., Kakavand, H. \& Mammen, J. The simplest solution to an underdetermined system of linear equations. IEEE Int. Symp. Inf. Theory - Proc.

618 15. Mustapha, A. \& Aris, A. Z. Multivariate statistical analysis and environmental 619 modeling of heavy metals pollution by industries. Polish J. Environ. Stud. 21, $620 \quad 1359-1367(2012)$.

621 16. Lee, J. Y., Ryu, S. H., Lee, G. \& Bae, G. N. Indoor-to-outdoor particle 622 623 concentration ratio model for human exposure analysis. Atmos. Environ. 127, 100-106 (2016). 
624 17. Huang, Y. et al. A multimedia fugacity model to estimate the fate and transport of polycyclic aromatic hydrocarbons (PAHs) in a largely urbanized area, Shanghai, China. Chemosphere 217, 298-307 (2019).

18. Wanghe, K., Guo, X., Luan, X. \& Li, K. Assessment of urban green space based on bio-energy landscape connectivity: A case study on Tongzhou District in Beijing, China. Sustain. 11, (2019).

19. Xia, Z. et al. Health risk assessment on dietary exposure to polycyclic aromatic hydrocarbons (PAHs) in Taiyuan, China. Sci. Total Environ. 408, 5331-5337 (2010).

20. Baidu. Baidu Qianxi. (2020). Available at: https://qianxi.baidu.com/2020/.

21. Li, Q., Kim, M., Liu, Y. \& Yoo, C. K. Quantitative assessment of human health risks induced by vehicle exhaust polycyclic aromatic hydrocarbons at Zhengzhou via multimedia fugacity models with cancer risk assessment. Sci.

22. Abdel-Shafy, H. I. \& Mansour, M. S. M. A review on polycyclic aromatic hydrocarbons: Source, environmental impact, effect on human health and remediation. Egypt. J. Pet. 25, 107-123 (2016).

23. Boström, C. E. et al. Cancer risk assessment, indicators, and guidelines for polycyclic aromatic hydrocarbons in the ambient air. Environ. Health Perspect. 110, 451-488 (2002).

24. Meng, K. et al. The causes of 'Vulnerable Regions' to Air Pollution in Winter in the Beijing-Tianjin-Hebei Region: A topographic-meteorological impact model based on adaptive emission constraint technique. Atmosphere (Basel). 10, 719 (2019).

25. Ishtiaq, J. et al. Atmospheric Polycyclic Aromatic Hydrocarbons (PAHs) at 

Urban Settings in Pakistan: Spatial Variations, Sources and Health Risks. Chemosphere 129811 (2021). doi:10.1016/j.chemosphere.2021.129811

651 26. Shen, B., Wu, J., Zhan, S. \& Jin, M. Residues of organochlorine pesticides 652 (OCPs) and polycyclic aromatic hydrocarbons (PAHs) in waters of the Ili-Balkhash Basin, arid Central Asia: Concentrations and risk assessment.

654 Chemosphere 273, 129705 (2021).

655

656 
658 Figure 1 | Research diagram of the MLUF and the multivariate interpretation \& 659 system analyses.

660 Figure 2 | The proposed systematic model calibration method for the multiple land-use 661 model.

662 Figure 3 | Location of the study area — downtown and the Tong Zhou district area of 663 Beijing.

664 Figure 4 | The selected model parameter in multiple land-use fugacity model via VIP 665 scores for each PAH.

666 Figure 5 | Estimated PAH transformation and fate inside the compartments, as 667 determined by the multiple land-use fugacity mode.

668 Figure 6 | Risk assessment of (a) human and (b) ecological system exposure to PAHs 669 in the study area.

670 Figure $7 \mid$ Spatial distributions of $\Sigma_{16} \mathrm{PAHs}$ caused ILCR of the study area based on 671 multiple land-use fugacity model.

672 Figure $8 \mid$ The dynamic variation of $\Sigma_{16}$ PAHs concentration during the COVID-19

673 pandemic in each compartment. (a) air, (b) water, (c) soil_agriculture (d) soil_forest (e)

674 soil_garden (f) sediment, (g) vegetation_agriculture, (h) vegetation_forest, (i) vegetation_garden, 675 and (j) organic film.

676 


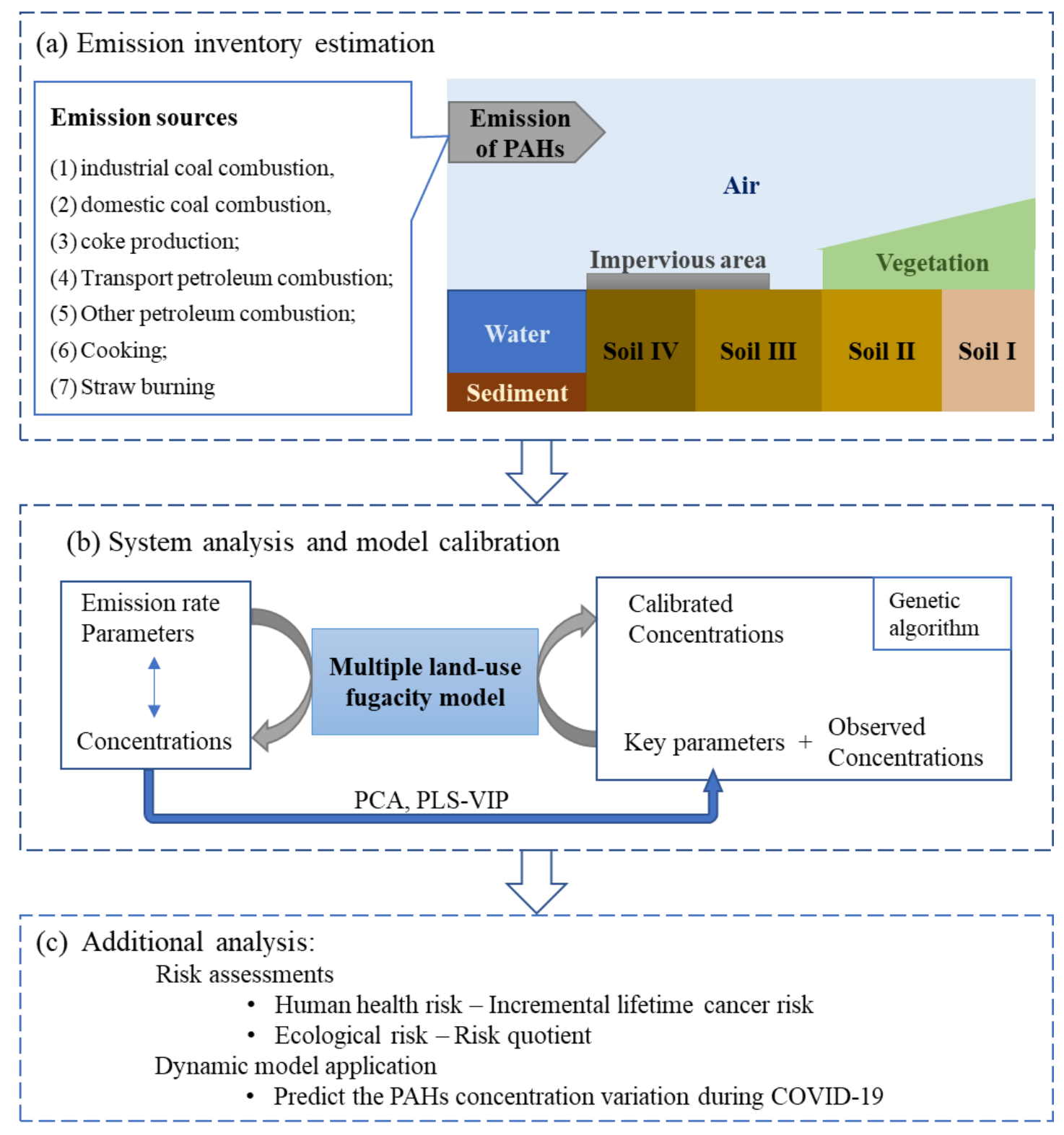

678 Figure $1 \mid$ Research diagram of the MLUF and the multivariate interpretation \& system 679 analyses. 


\begin{tabular}{|c|c|}
\hline \multirow{3}{*}{ Data collection } & $\begin{array}{l}\quad \text { Gathering the data } \\
\text { - Monte-Carlo simulation method } \\
\text { - Uniform distribution }\end{array}$ \\
\hline & $\begin{array}{l}\text { Data pre-processing } \\
\text { - Auto-scaling }\end{array}$ \\
\hline & 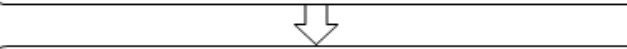 \\
\hline \multirow[t]{2}{*}{ System analysis } & $\begin{array}{l}\text { System analysis } \\
\text { - PCA and PLS methods }\end{array}$ \\
\hline & $\sqrt{3}$ \\
\hline Sensitivity analysis & $\begin{array}{l}\text { Key parameters selection } \\
\text { - } \quad \text { PLS-VIP scores } \\
\end{array}$ \\
\hline \multirow{3}{*}{ Optimization and calibration } & 5 \\
\hline & $\begin{array}{l}\text { Optimization } \\
\text { - Genetic algorithm }\end{array}$ \\
\hline & $\begin{array}{l}\text { Calibration } \\
\text { - Minimize the error between calculated and } \\
\text { observed concentration in each compartment }\end{array}$ \\
\hline \multirow[b]{2}{*}{ Model verification } & 25 \\
\hline & Check the model performances \\
\hline
\end{tabular}

681 Figure $2 \mid$ The proposed systematic model calibration method for the multiple land-use 682 model.

683

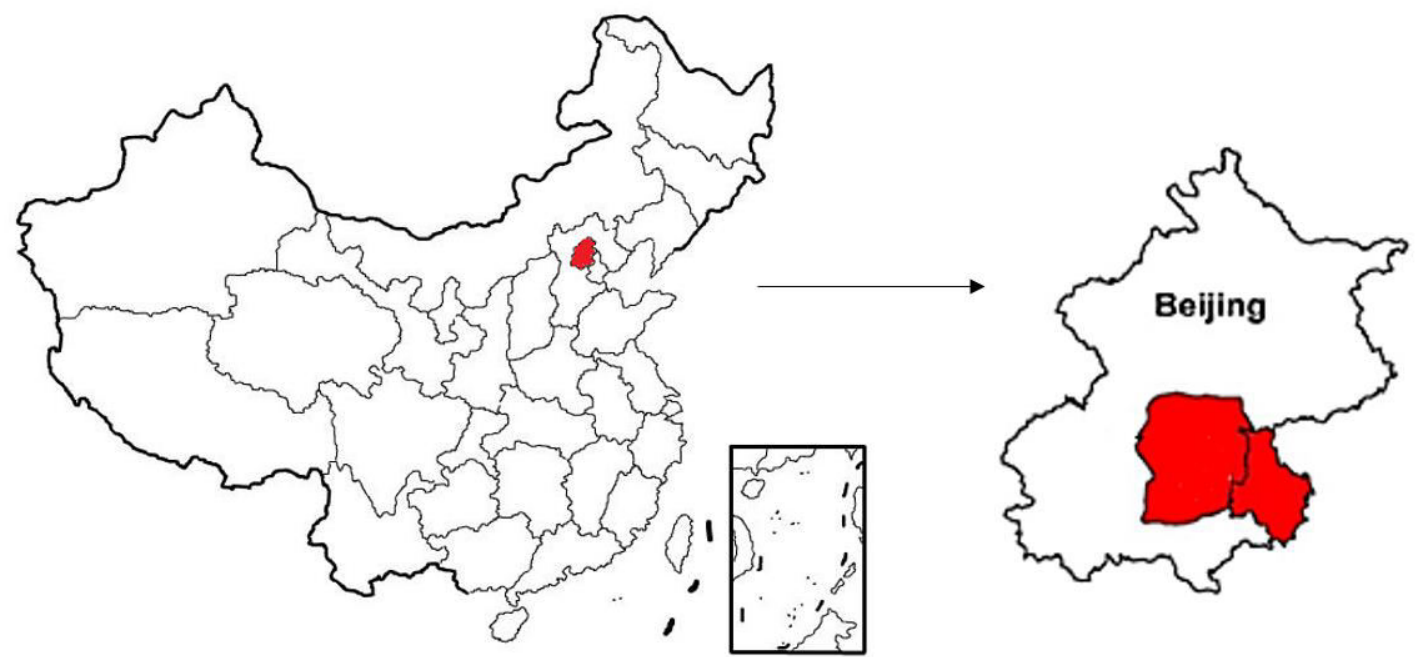

685 Figure 3 | Location of the study area — downtown and the Tong Zhou district area of 686 Beijing. 


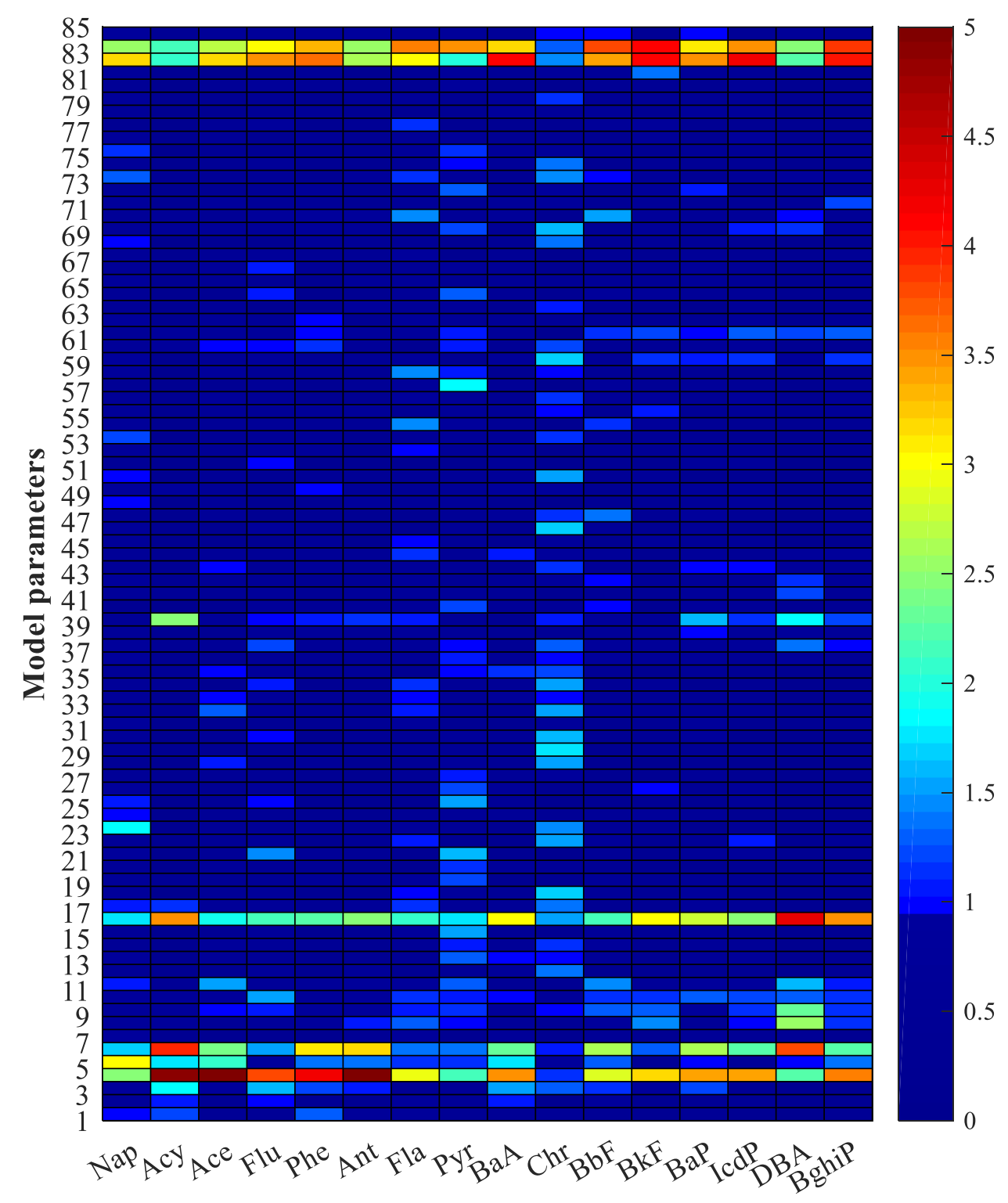

Polycyclic aromatic hydrocarbons

687

688 Figure $4 \mid$ The selected model parameter in multiple land-use fugacity model via VIP 689 scores for each PAH. 


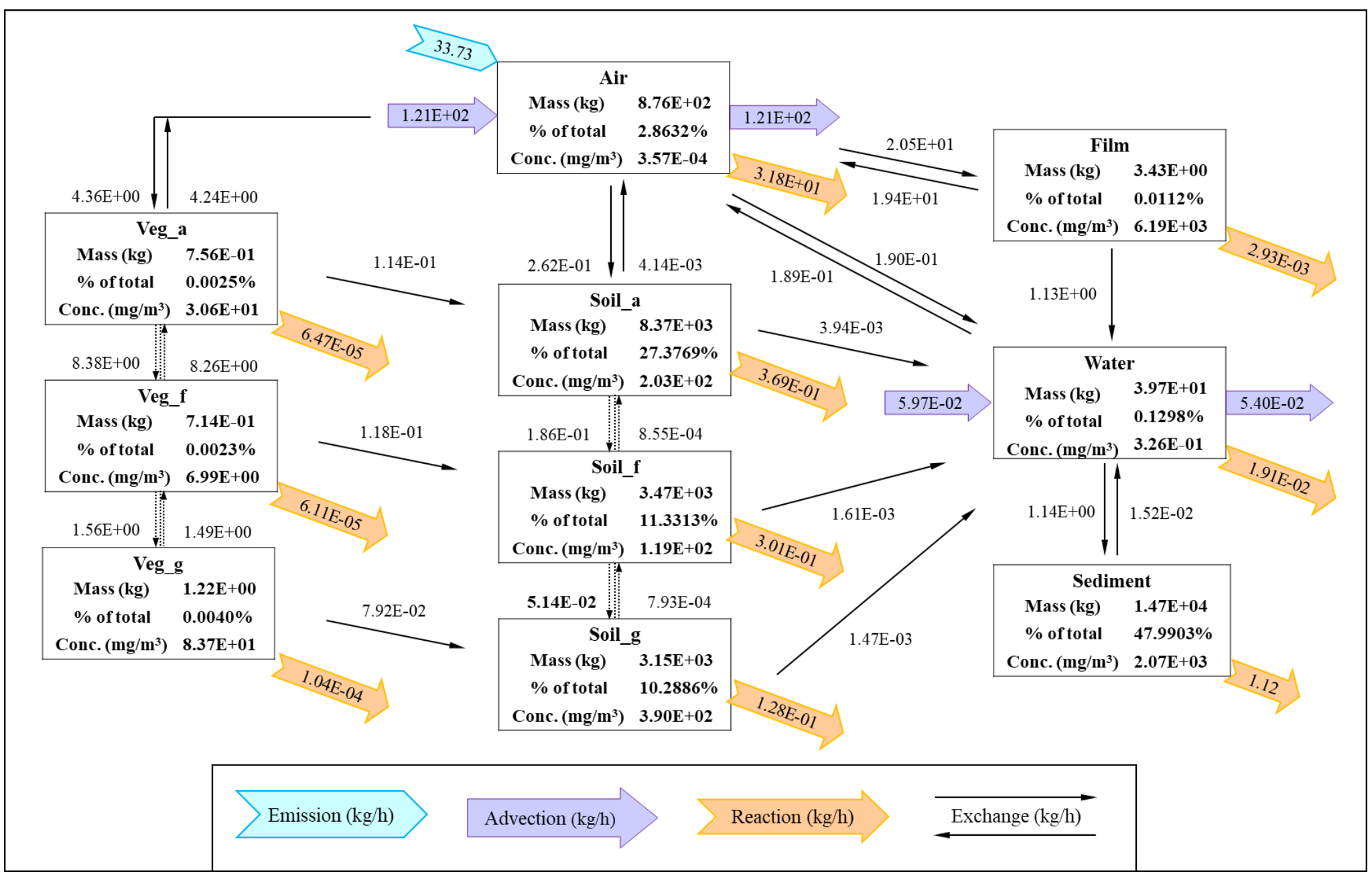

691 Figure 5 | Estimated PAH transformation and fate inside the compartments, as determined by the multiple land-use fugacity mode. 
692

693

694

695

696

697 in the study area.

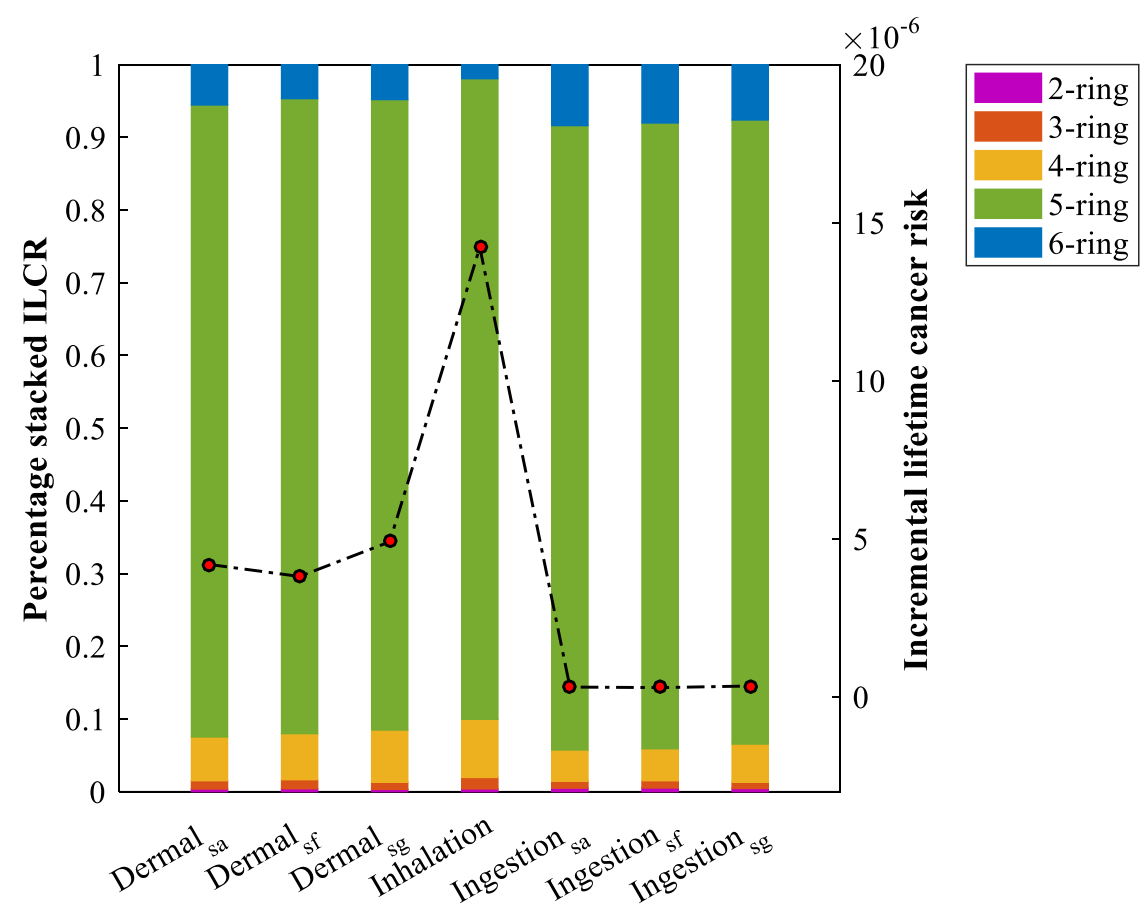

(a)

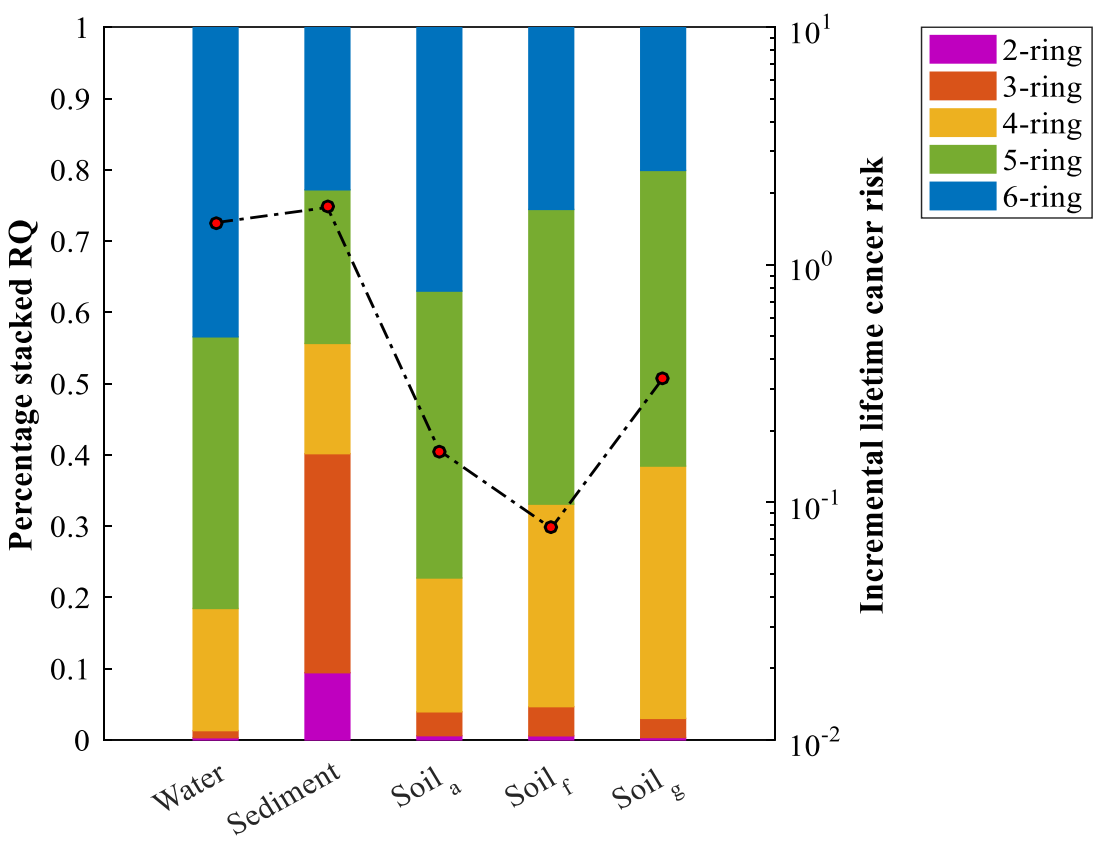

(b)

698

699 


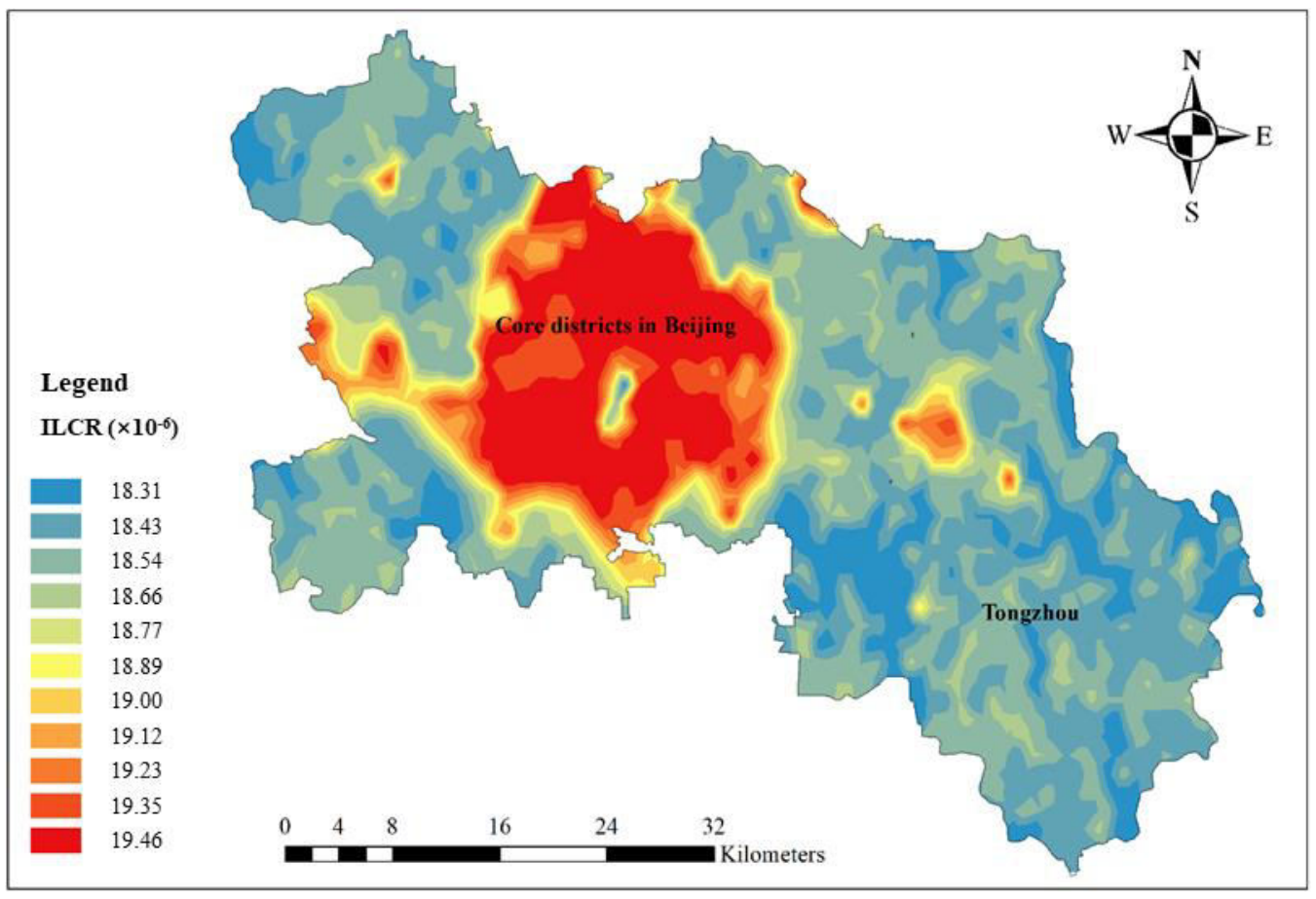

701 Figure 7 | Spatial distributions of $\Sigma_{16} \mathrm{PAHs}$ caused ILCR of the study area based on 702 multiple land-use fugacity model.

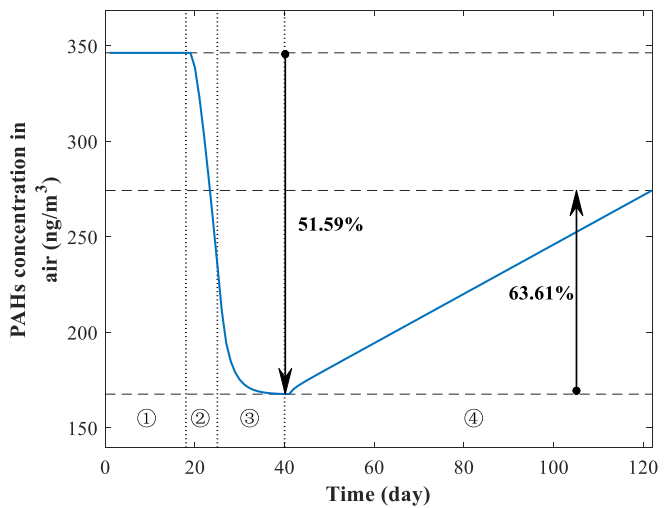

(a)

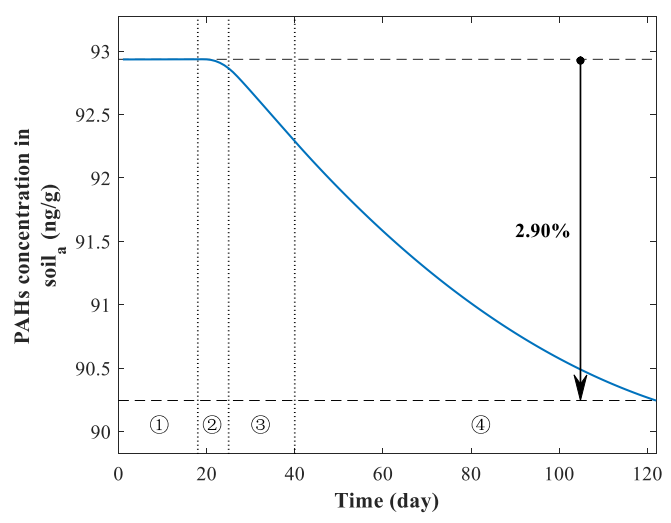

707

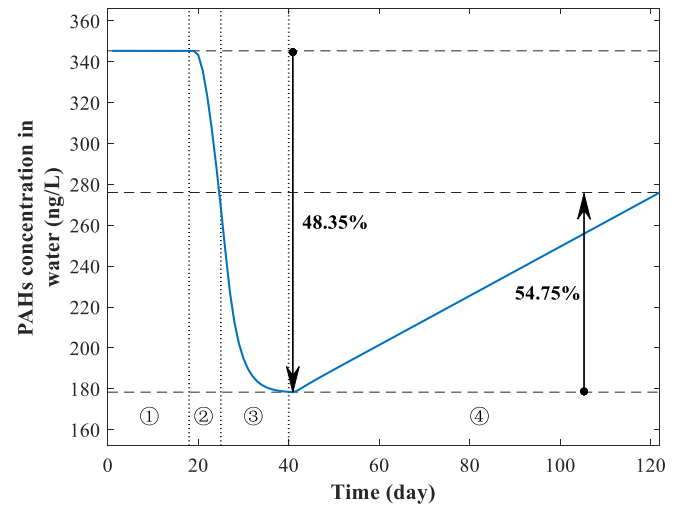

(b)

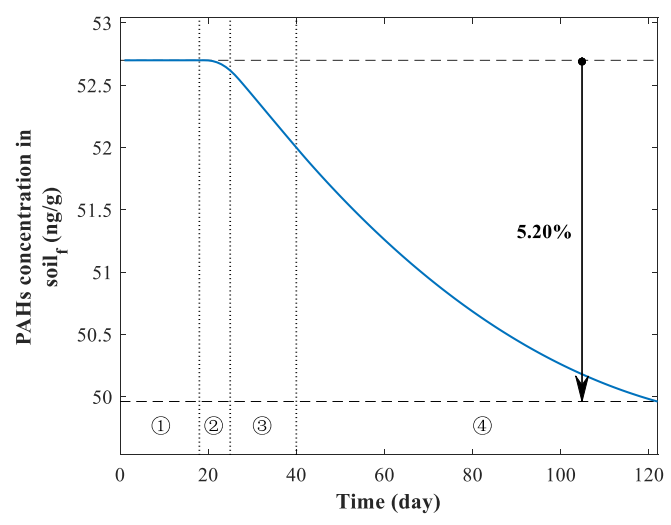

(d) 
708 709 710 711

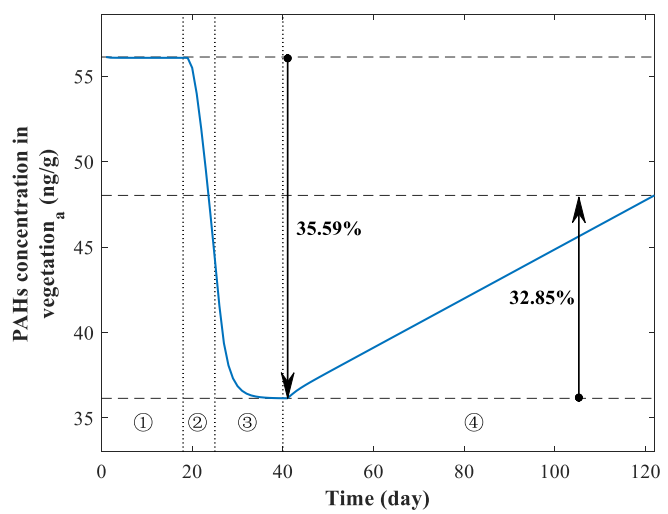

(g)

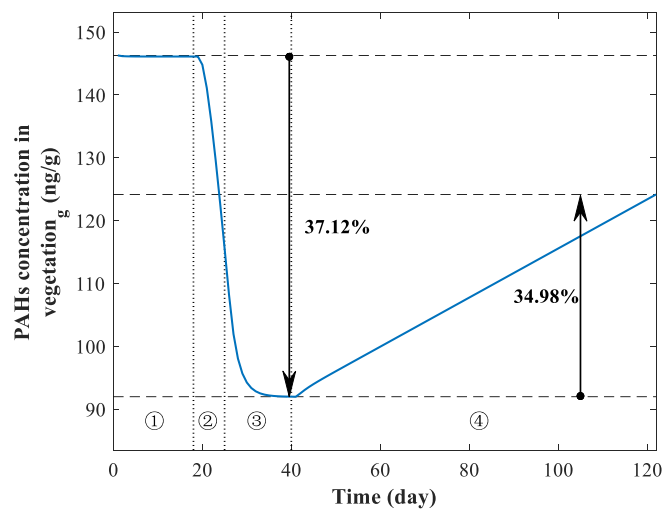

(i)

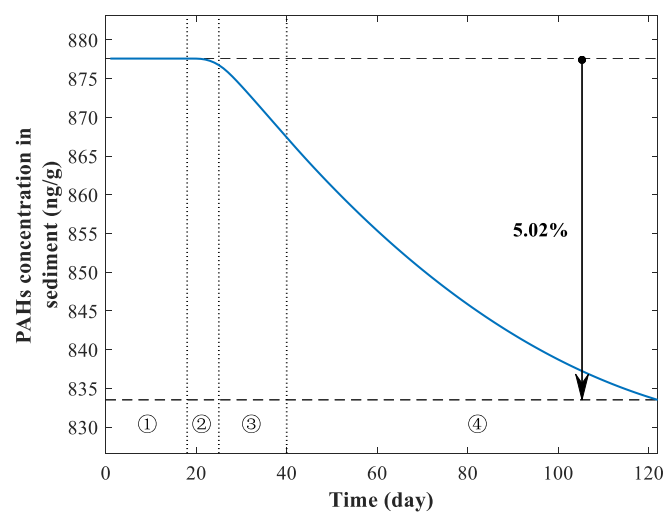

(f)

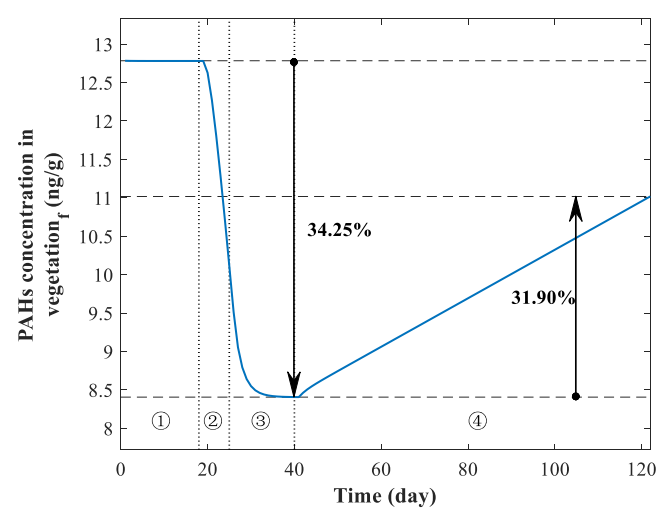

(h)

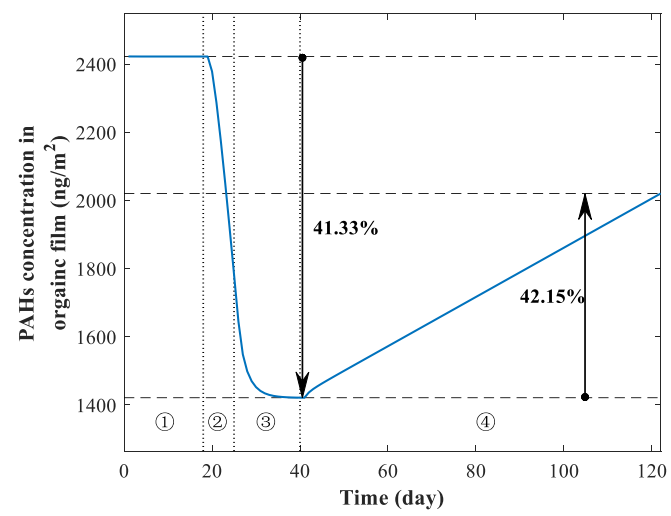

(j)

Figure 8 | The dynamic variation of $\Sigma_{16}$ PAHs concentration during the COVID-19 pandemic in each compartment. (a) air, (b) water, (c) soil_agriculture (d) soil_forest (e) soil_garden (f) sediment, (g) vegetation_agriculture, (h) vegetation_forest, (i) vegetation_garden, 
721 Table 1 | The mass balance equations for each compartment in MLUF model. 
Table 1 | The mass balance equations for each compartment in MLUF model.

\begin{tabular}{cc}
\hline Compartment & Mass balance equation \\
\hline Air & $E_{A}+\operatorname{Qin}_{A} C_{A}+D_{W A} f_{W}+\sum_{n=1}^{p}\left(D_{S n A} f_{S n}+D_{V n A} f_{V n}\right)+D_{F A} f_{F}$ \\
& $=f_{A}\left(D a_{A}+D r_{A}+D_{A W}+\sum_{i=1}^{n}\left(D_{A S n}+D_{A V n}\right)+D_{A F}\right)$ \\
Water & $E_{W}+Q \operatorname{Qin}_{W} C_{W}+D_{A W} f_{A}+\sum_{i=1}^{n}\left(D_{S n W} f_{S n}\right)+D_{S e W} f_{S e}+D_{F W} f_{F}$ \\
& $=f_{W}\left(D a_{W}+D r_{W}+D_{W A}+D_{W S e}\right)$ \\
Soils & $E_{S n}+D_{A S n} f_{A}+D_{V n S n} f_{V n}=f_{S n}\left(D r_{S n}+D_{S n A}+D_{S n W}\right)$ \\
Sediment & $D_{W S e} f_{W}=f_{S e}\left(D r_{S e}+D_{S e W}\right)$ \\
Vegetation & $D_{A V n} f_{A}=f_{V n}\left(D r_{V n}+D_{V n A}+D_{V n S n}\right)$ \\
Organic film & $D_{A F} f_{A}=f_{F}\left(D r_{F}+D_{F A}+D_{F W}\right)$
\end{tabular}




\section{Supplementary Files}

This is a list of supplementary files associated with this preprint. Click to download.

- SRSupplementarylnformation.docx 